Irish Math. Soc. Bulletin

Number 75, Summer 2015, 45-57

ISSN 0791-5578

\title{
ON THE CONTINUITY OF THE INVERSES OF STRICTLY MONOTONIC FUNCTIONS
}

\author{
HEIKO HOFFMANN
}

\begin{abstract}
In this short note we present an elementary, but seemingly not well known result on the continuity of the inverse of a strictly monotonic function and we discuss the relation of this result to the question when order and subspace topology are identical, both on the real line as well as in the abstract framework of connected linearly ordered spaces.
\end{abstract}

\section{INTRODUCTION.}

It is a fundamental question in analysis under which conditions the inverse of a continuous bijection, say between two topological spaces, is itself continuous. There are well-known results like the invariance of domain theorem or the classical (and easy to prove) result that the inverse of a continuous bijection from a compact space onto a Hausdorff space is also continuous; see also [5] for a complete characterisation of all subsets of $\mathbb{R}$ such that every continuous injection defined on a set of this kind is a homeomorphism onto its range.

It seems that results like the ones just mentioned have influenced the presentation of similar results at the level of undergraduate courses. So it seems that the following statement is most widespread in such courses.

If $\emptyset \neq I \subseteq \mathbb{R}$ is an interval and if $f: I \rightarrow \mathbb{R}$ is continuous and injective, then $f^{-1}: f(I) \rightarrow \mathbb{R}$ is continuous, too.

Usually, the proofs given for this result make use of the continuity of $f$ in such a way that the continuity assumption appears to be indispensable at a first cursory glance. However, there is a more general result (see, e.g., [4, 37.1]), which, unfortunately, seems to be seldom taught in undergraduate courses.

2010 Mathematics Subject Classification. 26A48.

Key words and phrases. continuous inverse, strictly monotonic function.

Received on 19-3-2015; revised 6-6-2015.

(C)2015 Irish Mathematical Society 
If $\emptyset \neq I \subseteq \mathbb{R}$ is an interval and if $f: I \rightarrow \mathbb{R}$ is strictly monotonic, then $f^{-1}: f(I) \rightarrow \mathbb{R}$ is continuous, too.

This statement demonstrates that the premise of the continuity of $f$ is entirely superfluous (of course, injectivity must be replaced by strict monotonicity) and proofs based on this premise might disguise the deeper reason for this phenomenon. In fact, from the point of view of topology, the true reason lies in the following observation (readers not very well familiar with abstract topology may skip the subsequent explanation at their first reading): a strictly monotonic function $f: I \rightarrow f(I)$ is a homeomorphism if $I$ and $f(I)$ both carry the order topology induced by the order inherited from $\mathbb{R}$ instead of the usual subspace topology. Since the subspace topology is finer than the order topology the mapping $f^{-1}: f(I) \rightarrow I$ is continuous if $f(I)$ is endowed with the subspace topology and $I$ carries the order topology. But since for intervals the order and subspace topology coincide, we conclude that $f^{-1}: f(I) \rightarrow I$ is continous where $I$ and $f(I)$ now both carry the usual subspace topology.

Clearly, the same argument works for every strictly monotonic function $f: A \rightarrow \mathbb{R}(\emptyset \neq A \subseteq \mathbb{R})$ whenever the order and subspace topology of $A$ coincide. Unfortunately, the above proof (no matter how simple it is) is in general out of reach for an undergraduate course due to the topological conceptual framework. So at this point three questions arise:

(1) Is there a simple (i.e., ideally so simple that it is easily accessible to undergraduate students with no knowledge of abstract topology) description of those subsets of $\mathbb{R}$ for which the order and subspace topology of $A$ coincide?

(2) Is there an elementary proof for the above statement about the continuity of the inverse of a strictly monotonic function defined on such a set?

(3) Does there exist a subset of $\mathbb{R}$ such that each strictly monotonic function defined on this set has a continuous inverse, but the subspace and order topology on this set are distinct?

In this note we answer the first two questions affirmatively and we present such an elementary proof, which might be easily incorporated into an undergraduate course. This proof is given in the next section, where we choose a formulation that completely avoids mention of the order topology and we get along only with notions easily accessible to undergraduate students. Furthermore, we shall show 
that this result is optimal in the sense that on each non-empty subset of $\mathbb{R}$ for which order and subspace topology differ there exists a strictly monotonic function whose inverse is not continuous, thus giving a negative answer to the third question.

In the last section we take up once again the abstract topologist's position in order to complete our picture and to relate Proposition 2.1 and Proposition 2.4 below to the topological point of view described above. This link is provided by Lemma 3.3, which in fact answers the first of the above questions (see Corollary 3.5).

\section{Strictly monotonic FUnCtions on subsets of $\mathbb{R}$}

In this section we do not want to presuppose that the reader is familiar with abstract topology in order to make sure that this part of the note is also readable, e.g., for undergraduate students. For this reason we first clarify some notions occuring in what follows.

The symbol $\mathbb{N}$ denotes the set of strictly positive integers, while $\mathbb{N}_{0}:=\mathbb{N} \cup \dot{\{0}$.

Let $A$ be a subset of the reals $\mathbb{R}$. A set $C \subseteq A$ is called a (connected) component of $A$ if $C$ is an interval (where we include the degenerate cases of the empty set and singletons) and if each interval $I \subseteq A$ containing $C$ already coincides with $C$. Each set $A$ is the disjoint union of all its connected components. This is most easily seen by defining an equivalence relation on $A$ by setting $a \sim a^{\prime}: \Longleftrightarrow$ $\left[\min \left\{a, a^{\prime}\right\}, \max \left\{a, a^{\prime}\right\}\right] \subseteq A$ for $a, a^{\prime} \in A$. Then the equivalence classes of $\sim$ are precisely the connected components of $A$.

We call $A$ an open set if for each $a \in A$ there exists $\varepsilon>0$ such that $(a-\varepsilon, a+\varepsilon) \subseteq A$.

We denote by

$\partial A:=\{x \in \mathbb{R}: \forall \varepsilon>0:(x-\varepsilon, x+\varepsilon) \cap A \neq \emptyset \neq(x-\varepsilon, x+\varepsilon) \backslash A\}$

the boundary of $A$. The set $A$ is closed if and only if $\partial A \subseteq A$. Notice that $A$ is closed if and only if $\mathbb{R} \backslash A$ is open.

A function $f: A \rightarrow \mathbb{R}$ is continuous at a point $a \in A$ if for every sequence $\left(a_{n}\right)_{n}$ in $A$ converging to $A$ the sequence $\left(f\left(a_{n}\right)\right)_{n}$ converges to $f(a)$. The function $f: A \rightarrow \mathbb{R}$ is continuous (on $A$ ) if it is continuous at each point of $A$.

Now we can state and prove the announced result on the continuity of the inverse of strictly monotonic functions. 
Proposition 2.1. Let $\emptyset \neq A \subseteq \mathbb{R}$ be a set such that every bounded component of $\mathbb{R} \backslash A$ is either closed or open. Furthermore, let $f$ : $A \rightarrow \mathbb{R}$ be a strictly monotonic function on $A$. Then the function $f^{-1}: f(A) \rightarrow \mathbb{R}$ is continuous.

Proof. We suppose that $f$ is strictly increasing (the case that $f$ is strictly decreasing can be treated in a similar way).

Let $y_{0} \in f(A)$ be arbitrary. We want to show that $f^{-1}: f(A) \rightarrow \mathbb{R}$ is continuous at $y_{0}$. For this purpose, let $\left(y_{n}\right)_{n}$ be an arbitrary convergent sequence in $f(A)$ with limit $y_{0}$. We then have to show that $\left(x_{n}\right)_{n}:=\left(f^{-1}\left(y_{n}\right)\right)_{n} \in A^{\mathbb{N}}$ converges to $x_{0}:=f^{-1}\left(y_{0}\right) \in A$.

It is easy to verify that there are $u, v \in f(A)$ with $u \leq v$ such that $y_{n} \in[u, v]$ for all $n \in \mathbb{N}_{0}$. We put $a:=f^{-1}(u)$ and $b:=f^{-1}(v)$. Then we have $x_{n} \in[a, b] \cap A$ for all $n \in \mathbb{N}_{0}$. In particular, the sequence $\left(x_{n}\right)_{n}$ is bounded and it thus suffices to verify that $x_{0}$ is its only possible limit point in order to conclude that $\left(x_{n}\right)_{n}$ converges to $x_{0}$, which completes the proof. Indeed, suppose to the contrary that $x_{0}$ is the only possible limit point of the sequence $\left(x_{n}\right)_{n}$, but this sequence does not converge to $x_{0}$. Then we may pass to a subsequence $\left(x_{n_{k}}\right)_{k}$ such that $\left|x_{n_{k}}-x_{0}\right| \geq r$ for all $k \in \mathbb{N}$ and some $r>0$. Since $\left(x_{n_{k}}\right)_{k}$ is bounded as well, it has an accumulation point thanks to the Bolzano-Weierstraß theorem, say $x_{0}^{\prime}$, and we deduce $\left|x_{0}^{\prime}-x_{0}\right| \geq r$, i.e., $x_{0}^{\prime} \neq x_{0}$ on the one hand. But on the other hand $x_{0}^{\prime}$ is also an accumulation point of the sequence $\left(x_{n}\right)_{n}$ itself and therefore $x_{0}=x_{0}^{\prime}$ by hypothesis and so we end up with a contradiction.

Suppose now that $\left(x_{n}\right)_{n}$ possesses a limit point $\xi$ different from $x_{0}$ and let $\left(x_{n_{k}}\right)_{k}$ be a subsequence converging to $\xi$. We then either have $\xi>x_{0}$ or $\xi<x_{0}$. We only treat the first case (the second one is analogous) and we shall show that we obtain a contradiction.

First, assume additionally that $\xi$ does not belong to $A$ and denote by $I$ that component of $\mathbb{R} \backslash A$ that contains $\xi$. Observe that we have $\xi \in \partial I$ because of $\xi \in \partial A$.

If $\xi$ is the left endpoint of $I$ and if $I$ is not a singleton, then there exists a $k_{0} \in \mathbb{N}$ with $x_{n_{k_{0}}} \in\left(x_{0}, \xi\right)$ and an index $k_{1} \in \mathbb{N}$ with $x_{n_{k}} \in\left(x_{n_{k_{0}}}, \xi\right)$ for all $k \geq k_{1}$. This yields

$$
y_{n_{k}}=f\left(x_{n_{k}}\right) \geq f\left(x_{n_{k_{0}}}\right)>f\left(x_{0}\right)=y_{0}
$$

for all $k \geq k_{1}$. As $k \rightarrow \infty$ we obtain the contradiction $y_{0} \geq f\left(x_{n_{k_{0}}}\right)>$ $y_{0}$. 
If $\xi$ is the right endpoint of $I$ (which includes the case that $I$ is a singleton), then $I$ must be bounded due to $x_{0}<\xi$. By assumption $I$ is either closed or open, but due to $\xi \in \partial I \cap(\mathbb{R} \backslash A)$, the set $I$ must be closed. Therefore we then have $I=[\alpha, \xi]$ with an $\alpha \leq \xi$ such that $\alpha \notin A$.

We may now choose an element $z \in\left(x_{0}, \alpha\right) \cap A$. (Note that this is indeed possible: If $\alpha<\xi$, this follows from $\alpha \in \partial A$ and $x_{0}<$ $\xi$, which yields $x_{0}<\alpha$. If however $\alpha=\xi$, then $\left(x_{0}, \xi\right) \cap A$ is nonvoid since otherwise we would obtain $\left(x_{0}, \xi\right] \subseteq I=\{\xi\}$, which is impossible.) There exists a $k_{0} \in \mathbb{N}$ such that $x_{n_{k}}>z$ for all $k \geq k_{0}$. This implies

$$
y_{n_{k}}=f\left(x_{n_{k}}\right) \geq f(z)>f\left(x_{0}\right)=y_{0}
$$

for all $k \geq k_{0}$ and we arrive at the contradition $y_{0} \geq f(z)>y_{0}$.

Summarizing, we infer that $\xi$ must be an element of $A$. Here we distinguish between two cases: $\left(x_{0}, \xi\right) \cap A \neq \emptyset$ or $\left(x_{0}, \xi\right) \cap A=\emptyset$. In the first case we choose $z \in\left(x_{0}, \xi\right) \cap A$ and proceed as in the above case where $\xi$ was a right endpoint of the above $I$ to arrive at a contradiction.

So let us assume that $\left(x_{0}, \xi\right) \cap A=\emptyset$. Then there exists a $k_{0} \in \mathbb{N}$ such that $x_{n_{k}} \geq \xi$ for every $k \geq k_{0}$. This yields $y_{n_{k}} \in[f(\xi), \infty)$ for each $k \geq k_{0}$, which leads to the contradiction $y_{0} \geq f(\xi)>f\left(x_{0}\right)=$ $y_{0}$.

Altogether we arrive at the conclusion that $\xi>x_{0}$ is not possible.

Proposition 2.1 gives rise to the following characterisation of the continuity of a strictly monotonic function.

Corollary 2.2. Let $\emptyset \neq A \subseteq \mathbb{R}$ such that every bounded component of $\mathbb{R} \backslash A$ is closed or open. Then for a strictly monotonic function $f: A \rightarrow \mathbb{R}$ the following assertions are equivalent.

(a) The function $f: A \rightarrow \mathbb{R}$ is continuous.

(b) Each bounded component of $\mathbb{R} \backslash f(A)$ is closed or open.

If either assertion holds, then the sets $A$ and $f(A)$ are homeomorphic. In particular, $f$ is continuous if its range $f(A)$ is closed, open or an interval. Moreover, the implication "(b) $\Longrightarrow(a)$ " is still true if we drop the assumption imposed on $A$.

Proof. Applying Proposition 2.1 to the function $f^{-1}: f(A) \rightarrow \mathbb{R}$ gives us the implication "(b) $\Longrightarrow(\mathrm{a})$ "; even without the assumption 
imposed on $A$.

Now assume that $f$ is continuous as well as, without loss of generality, that $f$ strictly increases. Furthermore, suppose to the contrary that $\mathbb{R} \backslash f(A)$ possesses a bounded component that is neither closed nor open, thus having the form $(u, v]$ or $[u, v)$. We only treat the first case.

Then $u \in f(A), v \notin f(A)$ and there is a strictly decreasing sequence $\left(y_{n}\right)_{n}$ in $f(A)$ with limit $v$. We set $x_{n}:=f^{-1}\left(y_{n}\right)$ for $n \in \mathbb{N}$ and $x:=f^{-1}(u)$. The sequence $\left(x_{n}\right)_{n}$ is strictly decreasing and bounded from below by $x$, thus it converges to $\xi:=\inf _{n \in \mathbb{N}} x_{n}$ in $\mathbb{R}$. The number $\xi$ does not belong to $A$ since otherwise the continuity of $f$ would imply

$$
v=\lim _{n \rightarrow \infty} y_{n}=\lim _{n \rightarrow \infty} f\left(x_{n}\right)=f(\xi) \in f(A),
$$

which is impossible because of $v \notin f(A)$. Now consider an arbitrary $z \in A$ with $z>x$. We then have $f(A) \ni f(z)>f(x)=u$ and thus $f(z)>v$. Consequently, there exists an index $n \in \mathbb{N}$ with $v<y_{n}=f\left(x_{n}\right)<f(z)$, which implies $\xi<x_{n}<z$. We conclude that $(x, \xi]$ is a component of $\mathbb{R} \backslash A$ (because $x \in A$ and $A \ni x_{n} \rightarrow \xi \notin A$ as $n \rightarrow \infty)$, which contradicts the assumption on $A$.

The first part of addendum is clear by Proposition 2.1.

Remark 2.3. The characterisation of the continuity of a strictly monotonic function obtained in the preceding corollary fails if the adverb "strictly" is dropped. Indeed, just consider the function $f:\left\{\frac{1}{n} ; n \in \mathbb{N}\right\} \cup\{0\} \rightarrow \mathbb{R}$ given by $f(0):=0$ and $f\left(\frac{1}{n}\right)=1(n \in \mathbb{N})$.

As announced we now demonstrate that Proposition 2.1 is in some sense optimal.

Proposition 2.4. Let $\emptyset \neq A \subseteq \mathbb{R}$ be a set such that $\mathbb{R} \backslash A$ possesses a bounded component that is neither closed nor open. Then there exists a strictly monotonic, continuous function $f: A \rightarrow \mathbb{R}$ such that the function $f^{-1}: f(A) \rightarrow \mathbb{R}$ is discontinuous.

Proof. By assumption $\mathbb{R} \backslash A$ possesses a bounded component having the form $(a, b]$ or $[a, b)$ (with $a<b$ ). We only consider the first case since the second case is analogous.

Clearly, $b$ is a cluster point of $(b, \infty) \cap A$. Therefore we can choose a strictly decreasing sequence $\left(x_{n}\right)_{n}$ in $A$ converging to $b$. Moreover, we choose a strictly decreasing sequence $\left(y_{n}\right)_{n}$ in $\mathbb{R}$ with limit $a$. 
Now we put $g\left(x_{n}\right):=y_{n}$ for $n \in \mathbb{N}$ and $g(a):=a$ and we extend $g$ on $\left(x_{n+1}, x_{n}\right)$ linearly. This gives us a strictly increasing, continuous function $g:\{a\} \cup\left(b, x_{1}\right] \rightarrow \mathbb{R}$, which we extend to a strictly increasing, continuous function $g:(-\infty, a] \cup(b, \infty) \rightarrow \mathbb{R}$ in any way. Then the function $f:=\left.g\right|_{A}$ (note that $A \subseteq(-\infty, a] \cup(b, \infty)$ ) is strictly increasing and continuous, but its inverse is discontinuous at $a$. In fact, we calculate $\lim _{n \rightarrow \infty} f\left(x_{n}\right)=\lim _{n \rightarrow \infty} y_{n}=a=f(a)$, while $\lim _{n \rightarrow \infty} f^{-1}\left(y_{n}\right)=\lim _{n \rightarrow \infty} x_{n}=b \neq a=f^{-1}(a)$.

Remark 2.5. (a) Proposition 2.1 and Proposition 2.4 together characterise those nonvoid subsets $A$ of $\mathbb{R}$ such that each (continuous) strictly monotonic function $f: A \rightarrow \mathbb{R}$ possesses a continuous inverse. These are precisely those non-empty sets $A$ such that all bounded connected components of $\mathbb{R} \backslash A$ are closed or open.

(b) By Proposition 2.1, the function $\left.g\right|_{(-\infty, a) \cup(b, \infty)}$ (where $g$ is as in the proof of Proposition 2.4) has a continuous inverse. Therefore the point $a$ is the only discontinuity of the above $f^{-1}$.

(c) Combined with the order topological considerations in the introduction, Proposition 2.4 furnishes a proof that the order and subspace topology of $A$ do not coincide whenever $\mathbb{R} \backslash A$ possesses a bounded component that is neither closed nor open. The converse is also true, see Corollary 3.5 below in the next section.

\section{Strictly monotonic FUnCtions on SUbSETS OF CONNECTED LINEARLY ORDERED SPACES}

In this section we want to go beyond the scope of real functions and embed the results of the preceding section into a more general framework in order to supply the topological background underlying these results. In particular, we want to explore whether there exists a reasonable generalisation of Proposition 2.1. As we shall see, it turns out that there is indeed a perfect analogue in the setting of connected linearly ordered spaces (see Proposition 3.7 below).

We presuppose from now on that the reader is acquainted with the most basic notions of abstract topology. Nevertheless we start by reviewing some important notions.

In what follows $\left(X, \leq_{X}\right)$ and $\left(Y, \leq_{Y}\right)$ are linearly ordered sets, both 
endowed with their respective order topology $\tau_{\left(X, \leq_{X}\right)}$ and $\tau_{\left(Y, \leq_{Y}\right)}$. If no confusion is to be expected, we drop the indices and simply write $\leq$

Let $\emptyset \neq A \subseteq X$. We may endow $A$ with two reasonable topologies: the subspace topology, denoted by $\tau_{A}$, generated by all sets of the form $(-\infty, x) \cap A$ or $(x, \infty) \cap A$ where $x \in X$, and the topology induced by the order on $A$ inherited from $X$, denoted by $\tau_{(A, \leq)}$, generated by all sets of the form $(-\infty, a) \cap A$ or $(a, \infty) \cap A$ where $a \in A$. We always have $\tau_{(A, \leq)} \subseteq \tau_{A}$, but this inclusion can be strict. Notice that $\tau_{(A, \leq)}=\tau_{A}$ if, for instance, $A$ is compact with respect to the subspace topology or an interval (see, e.g., [2, 4A2R (m)]).

We adopt the usual convention to write $(-\infty, x)$ resp. $(x, \infty)$ resp. $(-\infty, x]$ resp. $[x, \infty)$ instead of $\left\{x^{\prime} \in X: x^{\prime}<x\right\}$ resp. $\left\{x^{\prime} \in X\right.$ : $\left.x^{\prime}>x\right\}$ resp. $\left\{x^{\prime} \in X: x^{\prime} \leq x\right\}$ resp. $\left\{x^{\prime} \in X: x^{\prime} \geq x\right\}$. We write $\sup A=\infty$ if $A$ is not bounded from above and $\inf A=-\infty$ if $A$ is not bounded from below and by convention $-\infty<x<\infty$ for all $x \in X$ (even if $(X, \leq)$ has a minimum or maximum; in particular $\pm \infty \notin X)$.

Recall that $(X, \leq)$ is called Dedekind complete if every non-empty subset $A$ of $X$ with an upper bound has a least upper bound denoted by $\sup A$. If $(X, \leq)$ is Dedekind complete and $A$ is a nonvoid subset of $X$ with a lower bound, then $A$ possesses a greatest lower bound denoted by $\inf A$ (see, e.g., [1, 314B (b)]).

One says that $(X, \leq)$ is dense provided that for any two elements $x, x^{\prime} \in X$ with $x<x^{\prime}$ there exists an $x^{\prime \prime} \in X$ such that $x<x^{\prime \prime}<x^{\prime}$.

A subset $A$ of $X$ is called (order-) convex if for any two elements $a, a^{\prime} \in A$ the interval $\left[\min \left(a, a^{\prime}\right), \max \left(a, a^{\prime}\right)\right]$ is contained in $A$.

The next lemma collects some basic facts concerning order topologies, which are probably folklore. For this reason we omit the easy proofs (see also Exercise 26G in [7] for assertion (a))

Lemma 3.1. Let $(X, \leq)$ be a linearly ordered set endowed with the order topology and $\emptyset \neq A \subseteq X$.

(a) The space $X$ is connected if and only if $(X, \leq)$ is Dedekind complete and dense.

(b) If $A$ is connected w.r.t. $\tau_{A}$, then it is convex.

(c) If $A$ is an interval, then $A$ is convex. The converse is true provided that $(X, \leq)$ is Dedekind complete. 
(d) If $(X, \leq)$ is connected and $A$ is an interval, then $A$ is connected w.r.t. $\tau_{A}$.

(e) If $(X, \leq)$ is connected, then the set of subsets of $X$ that are connected (w.r.t. the subspace topology) coincides with the set of intervals.

Example 3.2. Subintervals of $\mathbb{R}$, the extended real line $\mathbb{R} \cup\{ \pm \infty\}$, the long line (see, e.g., counterexample 46 in [6]), the extended long line (see, e.g., counterexample 46 in [6]), the unit square with the lexicographical order (see, e.g., counterexample 48 in [6]) or lexicographic cubes (see section 2 of [3]) are examples for connected linearly ordered spaces $(X, \leq)$.

For a connected linearly ordered space $(X, \leq)$ the subsequent lemma provides a catchy characterisation of all subsets of $X$ for which the subspace and order topology coincide. Moreover, the following Lemma 3.3 (resp. Corollary 3.5) links Proposition 2.1 and Proposition 2.4 to the topological consideration from the introduction and completes our picture. In fact, using Lemma 3.3 we obtain a perfect generalisation of Proposition 2.1 as we shall see later on.

Lemma 3.3. Let $(X, \leq)$ be connected and $\emptyset \neq A \subseteq X$. Then the order and subspace topology of $A$ coincide if and only if every component of $X \backslash A$ w.r.t. the subspace topology $\tau_{A}$ is closed or open.

Proof. We first suppose that $X \backslash A$ possesses a component which is neither closed nor open. Thanks to part (e) of Lemma 3.1, there are $a, b \in X$ with $a<b$ such that either $[a, b)$ or $(a, b]$ is a component of $X \backslash A$. (Notice that all other kinds of intervals are surely closed or open.) We only treat the first case since the second one can be handled analogously.

We first observe that a cannot be the least element of $(X, \leq)$ (provided there exist any at all) because otherwise $[a, b)$ would be open. As a consequence, the set $(-\infty, a)$ is nonvoid. In addition, $(t, a) \cap A \neq \emptyset$ for each $t \in(-\infty, a)$. The latter assertion results from the fact that for a point $t \in(-\infty, a)$ with $(t, a) \cap A=\emptyset$ one would obtain $(t, b) \subseteq X \backslash A$. Since $(t, b)$ is connected by Lemma 3.1 (d) and $[a, b)$ is a connected component of $X \backslash A$ with $[a, b) \cap(t, b) \neq \emptyset$, we infer $(t, b) \subseteq[a, b)$. But as $(X, \leq)$ is dense, the set $(t, a)$ is nonempty. This yields $(-\infty, a) \cap[a, b) \neq \emptyset$, which is absurd. We now put $\mathcal{A}:=\{t \in X: t<a\}$, we let $\preceq$ denote the partial order $\leq$ on $\mathcal{A}$ and we choose $x_{t} \in(t, a) \cap A$ for each $t \in \mathcal{A}$. Then $(\mathcal{A}, \preceq)$ is an 
upwards directed (nonvoid) set and $\left(x_{t}\right)_{t \in \mathcal{A}}$ is a net in $A \cap(-\infty, a)$ that converges in $(X, \leq)$ to $a$, as one easily verifies. In particular, for every $x \in A$ with $x<b$, which implies $x<a$, resp. for each $x^{\prime} \in A$ with $x^{\prime}>b$, there is a $t_{0} \in \mathcal{A}$ with $x_{t} \in(x, \infty) \cap A$, resp. with $x_{t} \in\left(-\infty, x^{\prime}\right) \cap A$ for all $t \succeq t_{0}$. Therefore $\left(x_{t}\right)_{t \in \mathcal{A}}$ converges to $b$ with respect to the order topology on $A$.

If the order topology and the subspace topology of $A$ coincided, then we could infer that $\left(x_{t}\right)_{t \in \mathcal{A}}$ converges to $b$ with respect to the subspace topology on $A$, hence in $(X, \leq)$, which would yield $a=b$ (the order topology is always Hausdorff, see, e.g., [2, 4A2R (c)]) in contrast to $a<b$. As a result, the subspace topology of $A$ is strictly finer than the order topology of $A$. This establishes the only-if-part.

Now we conversely assume that each component of $X \backslash A$ is either closed or open. In order to show that in this case the order and subspace topology of $A$ are identical, it suffices to verify that each set of the form $(-\infty, \xi) \cap A$ or $(\xi, \infty) \cap A$, where $\xi \in X$, is open with respect to the order topology on $A$. We show this only for $(-\infty, \xi) \cap A$ because the remaining case can be treated similarly.

In the cases $\xi \in A,(-\infty, \xi) \cap A=\emptyset$ or $(-\infty, \xi) \cap A=A$ the assertion is clear. Therefore we may assume that $\xi \notin A$ and $(-\infty, \xi) \cap A \neq \emptyset$ and $(-\infty, \xi) \cap A \neq A$ or equivalently that $\xi \notin A$ and $(-\infty, \xi) \cap A \neq \emptyset$ and $(\xi, \infty) \cap A \neq \emptyset$. We denote by $I$ that component of $X \backslash A$ that contains $\xi$. Due to $(-\infty, \xi) \cap A \neq \emptyset$, $(\xi, \infty) \cap A \neq \emptyset$ and part (b) of Lemma 3.1, the set $I$ is bounded from above and from below. Thanks to Lemma 3.1 (e) the set $I$ is an interval and we thus deduce that there are $a, b \in X$ such that $I \in\{(a, b],[a, b),[a, b],(a, b)\}$.

We next show that none of the cases $I=[a, b)$ or $I=(a, b]$ can occur. We establish this claim only for the first case because an analogous argument works in the second one. The same argument as utilised above in the proof of the only-if-part gives us a net $\left(x_{t}\right)_{t \in \mathcal{A}}$ in $A \cap(-\infty, a)$ that converges in $(X, \leq)$ to $a$. (For this notice that $(-\infty, a)$ is non-empty because $A \cap(-\infty, \xi) \neq \emptyset$ and $[a, \xi] \subseteq X \backslash A$.) By hypothesis, $I$ is closed or open. If $I$ were open, then there would exist a $t_{0} \in \mathcal{A}$ such that $x_{t} \in I$ for all $t \succeq t_{0}$, which is impossible because of $(-\infty, a) \cap I=\emptyset$. Hence, $I$ is closed. Employing that $(X, \leq)$ is dense, one easily shows that $b$ is a cluster point of $I$, which yields $b \in I \subseteq X \backslash A$. But as $[a, b]$ is connected by Lemma 3.1 (d) 
and a strict superset of $I$, which is a connected component of $X \backslash A$, the point $b$ belongs to $A$. Contradiction!

Altogether we therefore either have $I=[a, b]$ with $a \leq \xi \leq b$ and $a, b \in X \backslash A$ or $I=(a, b)$ with $a<\xi<b$ and $a, b \in A$.

In the first case we can choose as before a net $\left(x_{t}\right)_{t \in \mathcal{A}}$ in $A \cap(-\infty, a)$ converging in $(X, \leq)$ to $a$. We then obtain

$$
(-\infty, \xi) \cap A=(-\infty, a) \cap A=\bigcup_{t \in \mathcal{A}}\left(\left(-\infty, x_{t}\right) \cap A\right),
$$

so that $(-\infty, \xi) \cap A$ is a union of sets open with respect to the order topology on $A$ and consequently itself open with respect to the order topology on $A$.

In the second case we observe that $[b, \infty) \cap A$ is closed with respect to the order topology on $A$ (because of $b \in A$ ). For this reason

$$
(-\infty, \xi) \cap A=A \backslash([b, \infty) \cap A)
$$

is open with respect to the order topology on $A$.

Example 3.4. (a) Lemma 3.3 applies to all closed subsets of a connected linearly ordered space $(X, \leq)$. Indeed, let $A$ be a closed subset of $X$. Then $X \backslash A$ is open and can be expressed as a union of disjoint open intervals (see, e.g., [2, 4A2R (j)]). Hence, each connected component of $X \backslash A$ is open.

(b) Furthermore, we may apply Lemma 3.3 to open subsets $U$ of a connected linearly ordered space $(X, \leq)$. To see this, note that each component of $X \backslash U$ is closed in $\left(X \backslash U, \tau_{X \backslash U}\right)$ because components of a topological space are always closed in this space. Hence, each component of $X \backslash U$ is closed in $(X, \leq)$ since $X \backslash U$ is closed in $(X, \leq)$.

(c) Lemma 3.3 also applies to each subset $D$ of a connected linearly ordered space $(X, \leq)$ which is dense in $X$. In fact, thanks to Lemma 3.1 every component of $X \backslash D$ is an interval. Since $(X, \leq)$ is dense, each non-empty interval that is not a singleton has nonvoid interior. Hence, every component of $X \backslash D$ is a singleton and consequently closed as $(X, \leq)$ is Hausdorff.

We record the following simple consequence of Lemma 3.3 .

Corollary 3.5. Let $\emptyset \neq A \subseteq \mathbb{R}$. Then the order and subspace topology of $A$ coincide if and only if every bounded component of $\mathbb{R} \backslash A$ is either closed or open. 
Remark 3.6. If we combine Proposition 2.1, Proposition 2.4 and Corollary 3.5, we arrive at the following result:

Let $\emptyset \neq A \subseteq \mathbb{R}$. Then the order and subspace topology of $A$ coincide if and only if every (continuous) strictly monotonic function $f$ : $A \rightarrow \mathbb{R}$ possesses a continuous inverse $f^{-1}: f(A) \rightarrow A$, where $A$ and $f(A)$ are endowed with their respective subspace topologies.

As previously promised we now arrive at the announced generalisation of Proposition 2.1.

Proposition 3.7. Let $\left(X, \leq_{X}\right)$ and $\left(Y, \leq_{Y}\right)$ be two linearly ordered spaces, where $\left(X, \leq_{X}\right)$ is connected. Let $A$ be a nonvoid subset of $X$ such that each component of $X \backslash A$ (w.r.t. the subspace topology $\left.\tau_{A}\right)$ is closed or open. Assume that $f: A \rightarrow Y$ is an injective orderpreserving or injective order-reversing mapping. Then the inverse mapping $f^{-1}:\left(f(A), \tau_{f(A)}\right) \rightarrow\left(A, \tau_{A}\right)$ is continuous.

Proof. If $f$ is order-reversing, then we define another total order $\leq_{Y}^{r}$ on $Y$ via

$$
y \leq_{Y}^{r} y^{\prime}: \Longleftrightarrow y \geq_{Y} y^{\prime}
$$

for $y, y^{\prime} \in Y$. Then $f:\left(A, \leq_{X}\right) \rightarrow\left(Y, \leq_{Y}^{r}\right)$ is order-preserving and it is not hard to verify that $\tau_{\left(Y, \leq_{Y}\right)}=\tau_{\left(Y, \leq_{Y}^{r}\right)}$. For this reason we may and will assume w.l.o.g. that $f$ is order-preserving. Then

$$
f:\left(A, \tau_{\left(A, \leq_{X}\right)}\right) \rightarrow\left(f(A), \tau_{\left(f(A), \leq_{Y}\right)}\right)
$$

is a homeomorphism. Because of $\tau_{\left(f(A), \leq_{Y}\right)} \subseteq \tau_{f(A)}$ and $\tau_{\left(A, \leq_{X}\right)}=\tau_{A}$ (the latter assertion results from Lemma 3.3 and the hypothesis), we conclude that

$$
f^{-1}:\left(f(A), \tau_{f(A)}\right) \rightarrow\left(A, \tau_{A}\right)
$$

is continuous as claimed.

We close this note with the following Question:

Does also an analogue of Proposition 2.4 hold in all connected linearly ordered spaces? Or to put it another way: Is an analogon of the characterisation obtained in Remark 3.6 valid in every connected linearly ordered space?

\section{REFERENCES}

[1] D. H. Fremlin, Measure Theory - Volume 3, Torres Fremlin, Colchester, 2004.

[2] D. H. Fremlin, Measure Theory - Volume 4, Torres Fremlin, Colchester, 2006. 
[3] R. G. Haydon, J. E. Jayne, I. Namioka, C. A. Rogers, Continuous functions on totally ordered spaces that are compact in their order topologies, J. Funct. Anal. 178 (2000), 23-63.

[4] H. Heuser, Lehrbuch der Analysis - Teil 1, 13th ed., B. G. Teubner, Stuttgart, Leipzig, Wiesbaden, 2000.

[5] M. J. Hoffman, Continuity of inverse functions, Math. Mag. 48 (1975), 6673.

[6] L. A. Steen and J. A. Seebach Jr., Counterexamples in Topology, Holt, Rinehart and Winston, Inc., New York-Montreal, Que.-London, 1970.

[7] S. Willard, General Topology, Addison-Wesley Publishing Co., Reading, Mass.-London-Don Mills, Ont., 1970.

Heiko Hoffmann received his Bachelor and Master degree from Saarland University in 2008 and 2010, respectively. He has recently completed his PhD thesis at the Karlsruhe Institute of Technology (KIT).

Department of Mathematics, Institute for Analysis, Karlsruhe Institute of Technology (KIT), 76128 Karlsruhe, Germany

E-mail address: heiko.hoffmann@kit.edu 